\title{
noRANSAC for fundamental matrix estimation
}

\author{
Fabio Bellavia \\ fabio.bellavia@unipa.it \\ Domenico Tegolo \\ domenico.tegolo@unipa.it
}

\author{
Department of Mathematics \\ and Computer Science \\ University of Palermo \\ Palermo, Italy
}

\begin{abstract}
The estimation of the fundamental matrix from a set of corresponding points is a relevant topic in epipolar stereo geometry [10]. Due to the high amount of outliers between the matches, RANSAC-based approaches [7, 13, 29] have been used to obtain the fundamental matrix. In this paper two new contributes are presented: a new normalized epipolar error measure which takes into account the shape of the features used as matches [17] and a new strategy to compare fundamental matrices. The proposed error measure gives good results and it does not depend on the image scale. Moreover, the new evaluation strategy describes a valid tool to compare different RANSAC-based methods because it does not rely on the inlier ratio, which could not correspond to the best allowable fundamental matrix estimated model, but it makes use of a reference ground truth fundamental matrix obtained by a set of corresponding points given by the user.
\end{abstract}

\section{Introduction}

In epipolar stereo geometry, the fundamental matrix $\mathrm{F}$ is a mathematical object which embeds the relation between corresponding points [10]. The computation of the fundamental matrix is a crucial task in many computer vision applications, such as three-dimensional reconstruction [27]. In order to provide its estimation, a relative small initial set of corresponding matches is required. This set is typically obtained by comparing image features extracted by a keypoint detector [17] according to a similarity measure obtained by a feature descriptor [16]. Though a lot of work has been done in this area, outliers represent a high portion of the initial matches used to compute the fundamental matrix and they can dramatically influence the final result. In order to alleviate this issue robust estimation algorithms have to be used, whose RANSAC (RANdom SAmple Consensus) [7] is the most popular.

The fundamental matrix model $\mathrm{F}$ is estimated from a random-chosen minimal set of matches, which reduces the change of the model to be contaminated by outliers, and it is tested against the whole set of matches according to any defined error measure. The best F so far is retained after each iteration, and the computation ends after a fixed number of iterations or until the probability to get a better model is low. The best inlier set is used to compute the final fundamental matrix [10].

Different approaches have been proposed to enhance RANSAC by improving the cost function [11, 29] and the sampling strategy [3, 4, 13, 15, 18, 19, 23, 25], or to deal with 
degenerate configurations [5, 8] and allow faster model checks [14, 20, 22], but also other similar approaches exist $[6,24,31]$.

In order to compare different RANSAC-based strategies, the computational efficiency and the correctness of model are considered. For the latter measure, the inlier ratio is usually adopted in the case on non-synthetic data [13, 25, 29]. However it does not always coincide with the most appropriate one, though it is the optimization function RANSAC tries to maximize, as in the case of scenes which contain a dominant plane or when error cost functions are not directly comparable. On the other side, to check correct matches by user inspection can be difficult in the case of huge amount of matches and it can be subjective, too.

The direct comparison between the correct and estimated fundamental matrices has been also proposed, however it does not provide a valid error measure [30], while sampling distances between the true and the estimated corresponding epipolar lines can provide good evaluations [30].

In this paper, two contributions are presented. A new normalized epipolar error measure to select inliers to estimate the fundamental matrix is described in Section 2, which takes into the account the shape of the features and does not introduce any further relevant computation. Moreover, it does not depend on the image scale, due to the normalization, and increases the model estimation.

Lastly, a new method to compare the fundamental matrix is provided in Section 4. It does not rely on the input matches but indirectly employs a user-provided ground truth fundamental matrix, which can be obtained easily by a small number of hand-taken matches. Evaluation results are discussed in Section 3 and final conclusions are reported in Section 5.

\section{The new normalized epipolar error}

Given a stereo image pair $\left(I_{1}, I_{2}\right)$ and corresponding points $\mathbf{x}_{1} \in I_{1}, \mathbf{x}_{2} \in I_{2}$ in homogeneous coordinates, the fundamental matrix $F$ determines the relation $\mathbf{x}_{2}^{\mathrm{T}} \mathrm{F} \mathbf{x}_{1}=0$. Geometrically the point $\mathbf{x}_{1}$ is constrained to lie on the epipolar line $\mathbf{l}_{1}=\mathbf{x}_{2}^{\mathrm{T}} \mathrm{F}$, and in similar way $\mathbf{x}_{2}$ on $\mathbf{l}_{2}=\mathbf{x}_{1}^{\mathrm{T}} \mathrm{F}^{\mathrm{T}}$. Epipolar lines pass through the epipoles $\mathbf{e}_{1} \in I_{1}, \mathbf{e}_{2} \in I_{2}$, which are respectively the right, left null-space of $\mathrm{F}[10]$.

The fundamental matrix F can be extracted by 8 or more correct correspondences by using the eight-point algorithm [10]. More matches are provided, better will be the estimation F, but the presence of outliers can strongly affected it [10]. Furthermore, it was shown in [21] that it is possible to obtain from 3 up to 10 feasible fundamental matrix solutions when 7 to 5 corresponding points are used respectively.

In order to estimate the goodness of the model, RANSAC tries to maximize the function $\xi$ of the number of correspondences for which the error $\varepsilon$ is less than the threshold value $t$ [7]

$$
\xi(\varepsilon) \begin{cases}1 & \text { if } \varepsilon<t \\ 0 & \text { otherwise }\end{cases}
$$

while MSAC (M-estimator SAC) [29] saturates the error up to the threshold value $t$ and a mixture between a Gaussian distribution for inliers and a uniform distribution for outliers is used in MLESAC (Maximum Likehood Estimator SAC) [29]. The Sampson error [10] is usually chosen as error measure $\varepsilon$, but also the distance $d$ of a point $\mathbf{x}$ to the corresponding epipolar line $\mathbf{l}$

$$
d(\mathbf{x}, \mathbf{l})=\left|\frac{\mathbf{l} \mathbf{x}}{(\mathbf{x})_{3}}\right|\left((\mathbf{l})_{1}^{2}+(\mathbf{l})_{2}^{2}\right)^{-\frac{1}{2}}
$$


where $(\mathbf{a})_{k}$ is the $k$-th component of the vector $\mathbf{a}$, that is the epipolar distance $d\left(\mathbf{x}_{i}, \mathbf{l}_{i}\right)$. Slightly better results have been reported with the former error measure [10].

Let $\mathscr{R}_{1} \in I_{1}, \mathscr{R}_{2} \in I_{2}$ be two elliptical feature patches centred in $\mathbf{x}_{1}, \mathbf{x}_{2}$ as commonly extracted by feature detectors [17], with minor and major axes respectively $\alpha_{\min _{i}}, \alpha_{\max _{i}}$, $i \in\{1,2\}$. As done for the feature descriptor computation [16], the scale is increased by a factor of three to include the boundary data.

The error measure $\kappa_{i}$ in the image $I_{i}$, for the feature pair $\left(\mathscr{R}_{1}, \mathscr{R}_{2}\right)$ is defined as

$$
\kappa_{i}=\min \left(\frac{d\left(\mathbf{x}_{i}, \mathbf{l}_{i}\right)}{\alpha_{\min _{i}}}, 1\right)
$$

that is, the epipolar distance $d\left(\mathbf{x}_{i}, \mathbf{l}_{i}\right)$ between the feature centre $\mathbf{x}_{i}$ and its epipolar line $\mathbf{l}_{i}$, computed by using the corresponding point in the other image, is normalized by the minor axis of the feature ellipse $\mathscr{R}_{i}$.

The error $\kappa_{i}$ achieves the maximal value of 1 roughly when the supposed reprojected feature ellipse would not touch the actual ellipse, as shown in Figure 1. Clearly, when accidentally a wrong feature $\mathscr{R}_{i}$ lies close to the correct epipolar line $\mathbf{l}_{i}$, the error $\kappa_{i}$ is misleading, as it also happens for both the Sampson error and the epipolar distance $d\left(\mathbf{x}_{i}, \mathbf{l}_{i}\right)$. To take into the account the possible error committed by the keypoint detector in the extraction of the feature $\mathscr{R}_{i}$, the minor axis $\alpha_{\text {min }_{i}}$ is preferred to the major axis $\alpha_{\max }$ in the normalization, i.e. a more pessimistic assumption is made for the worst case scenario. However, since the feature axis ratio is usually related to the intensity variation of the keypoint region in all directions, which detectors often try to maximize [2, 17], the values of $\alpha_{\max _{i}}$ and $\alpha_{\text {min }_{i}}$ are usually close.

The proposed error $\kappa_{i}$ does not depend on the image scale and provides a soft threshold $t$ on $\xi$, thus a possible overfitting on matches derived by a non-optimal choice of $t$ can be alleviated.

About the complexity of the normalized epipolar error $\kappa_{i}$ with respect to the standard epipolar distance $d\left(\mathbf{x}_{i}, \mathbf{l}_{i}\right)$, only a negligible overhead is added for computing in advance the minor axis length for each feature involved in the matching, plus the time required by a division and a max operation at runtime.
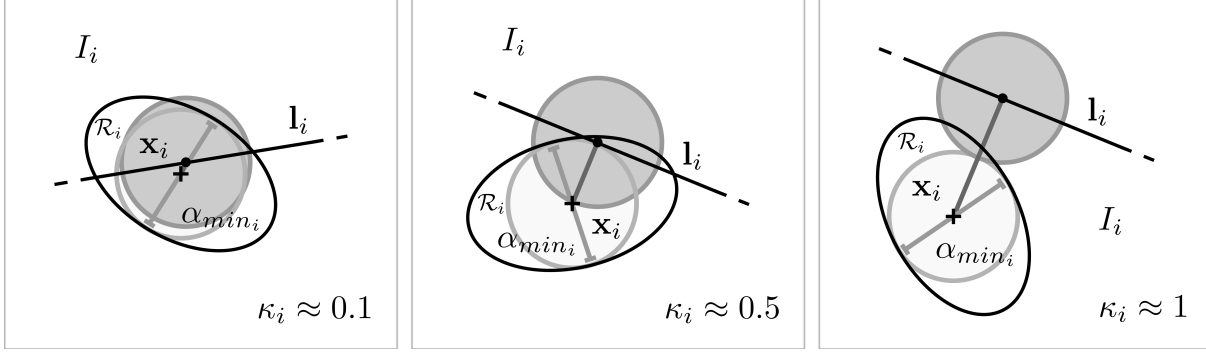

Figure 1: Examples of different values of the normalized epipolar distance $\kappa_{i}$. The dark grey circle represents the reprojected feature supposed by the error measure $\kappa_{i}$, the light grey circle is the approximation of the feature ellipse $\mathscr{R}_{i}$. The epipolar distance $d\left(\mathbf{x}_{i}, \mathbf{l}_{i}\right)$ is given by the dark grey segment joining the centres of the two circles and the minor axis $\alpha_{\text {min }_{I}}$ is shown as the light grey segment. 


\section{Proposed evaluation strategy}

\subsection{Motivations}

In order to compare fundamental matrices estimated by different algorithms on non-synthetic data, the inlier ratio is commonly adopted [13, 25, 29]. Although the maximization of the number of inliers coincides with the formulation of the optimization problem used by RANSAC-based approaches, i.e. find the best F compatible with the largest input dataset, this does not always correspond to the desired real solution. To test different algorithms in the case of the computation of the fundamental matrix, it can be easily seen that the threshold $t$ should remain fixed. By increasing the threshold, a large consensus set of points is usually found which could wrongly lead to include outliers. On the other side, by choosing a lower threshold value $t$ correct inliers can be discarded and no sufficient data could be present to get a good estimate F. A reasonable value of $t$ which should be maintained fixed among the different algorithms have to be used, but it cannot always be adopted when the error measure are not commensurable. This is the case of a comparison between the epipolar distance $d\left(\mathbf{x}_{i}, \mathbf{l}_{i}\right)$ expressed in pixel and the new proposed normalized epipolar error $\kappa_{i}$ which ranges in $[0,1]$.

Furthermore, the theoretical best model obtained by a robust estimator algorithm, could not meet the correct solution even if a prefixed value of $t$ is used. It can happen for instance in some degenerate cases when a dominant plane is present as depicted in Figure 2, where the initial set of matches (see Figure 2 (e)) contains an high fraction of outliers (red squares). Although a higher number of putative inliers is found by the model (see Figure 2 (a-b)), which is clearly wrong, the true correct fundamental matrix presents less true inliers (see Figure 2 (c-d)).

Moreover, it was shown that a direct numerical comparison between fundamental matrices by the Frobenius norm does not provide a valid error measure [30], while sampling distances between the true and the estimated corresponding epipolar lines can characterize the error between the matrices [30].

\subsection{The proposed approach}

In order to deal with these issues a new relation between lines on the image is defined. Given two lines $\mathbf{r}, \mathbf{s} \in \mathbb{R}^{2}$, let $\mathscr{C}_{\mathbf{r}, \mathbf{s}}$ be the cone obtained by intersecting the two half-planes given by $\mathbf{r}$ and $\mathbf{s}$ so that the minimum intersection angle is considered, as shown in Figure 3 (a), and the resulting surface is not null in the case of parallel lines as in Figure 3 (b). When the intersection angle is $\pi / 2$, any of the possible cones can be choose without distinction.

The intersection area $l_{I}$ of the image surface $I$ and $\mathscr{C}_{\mathbf{r}, \mathbf{s}}$, normalized on the whole image area surface is defined as

$$
l_{I}(\mathbf{r}, \mathbf{s})=\frac{A\left(I \cap \mathscr{C}_{\mathbf{r}, \mathbf{s}}\right)}{A(I)}
$$

where $A(\cdot)$ defines the surface area. The $l_{I}$ ratio can be seen as the minimum amount of work inside the image $I$ needed to move a line to the other, with $l_{I} \approx 0$ when the lines are almost the same.

Let $\mathrm{F}, \mathbf{l}_{i}$ be the true fundamental matrix and the true epipolar line corresponding to the point $\mathbf{x}_{i} \in I_{i}, i \in\{1,2\}$, given the stereo pair $\left(I_{1}, I_{2}\right)$, and $\widetilde{\mathrm{F}}, \widetilde{\mathbf{l}}_{i}$ as their estimation respectively. 


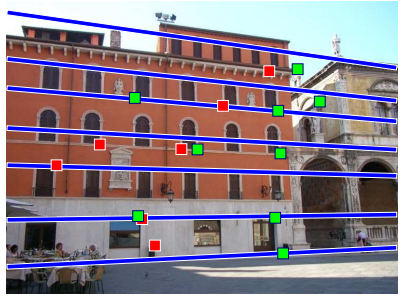

(a)

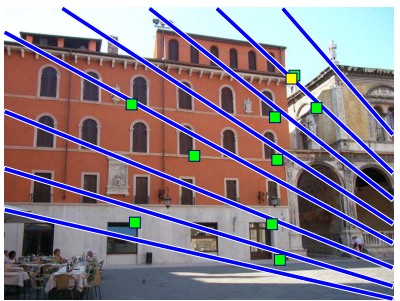

(c)

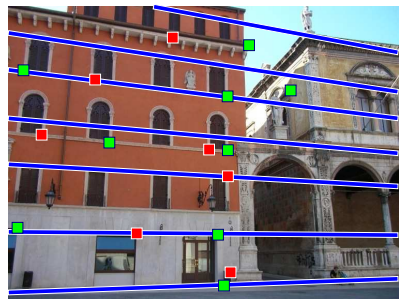

(b)

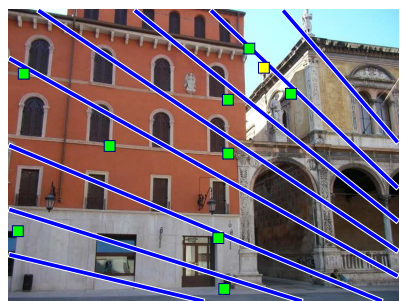

(d)

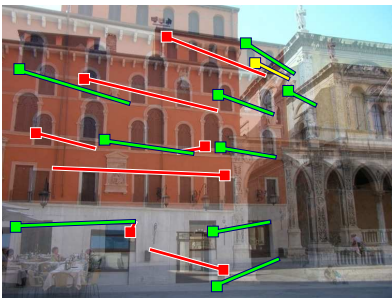

(e)

Figure 2: An example for which the best theoretical model obtained by RANSAC (a-b) does not coincide with the real solution (c-d). In both cases, epipolar lines (blue lines) are shown as well as inliers (squares). The initial set of matches (e) contains correct matches (green, yellow squares) and wrong matches (red squares). A correct match (yellow square) is missed in the bad model with an inlier ratio of $95 \%$, opposing to the correct solution for which it is $63 \%$.

For a point $\mathbf{x}_{i} \in I_{i}$, the error

$$
\begin{gathered}
\varphi_{i}\left(\mathbf{x}_{i}\right)=l_{I_{\bar{i}}}\left(\mathbf{x}_{i}^{T} \mathrm{~F}_{i}, \mathbf{x}_{i}^{T} \widetilde{\mathrm{F}}_{i}\right)=l_{I_{\bar{i}}}\left(\mathbf{l}_{\bar{i}}, \widetilde{\bar{i}}_{\bar{i}}\right) \\
\text { with } \quad \bar{i}=3-i, \quad \mathrm{~F}_{i}= \begin{cases}\mathrm{F} & \text { if } i=1 \\
\mathrm{~F}^{\mathrm{T}} & \text { otherwise }\end{cases}
\end{gathered}
$$

is an indirect measure between the matrices $F$ and $\widetilde{F}$ on the stereo image pair, which can be seen as a dense generalization of the measure proposed in [30]. For each point $\mathbf{x}_{i}$ on the image $I_{i}$, the epipolar lines $\mathbf{I}_{\bar{i}}$ and $\widetilde{\mathbf{l}}_{\bar{i}}$ are determined on the other image $I_{\bar{i}}$, defining a map $\varphi_{i}$ which is almost continuous on the image, as shown in Figure 4, and defines a fingerprint of the difference between the matrices. Discontinuities can happen by switching the cone surface near a $\pi / 2$ intersection angle, but only for high error models (see Figure 4 (d-e)). The error measure $\varphi_{i}$ can be low for an high image portion, because for a finite epipole $\mathbf{e}_{i}$ and its estimation $\widetilde{\mathbf{e}}_{i}$ the corresponding epipolar line pencils share a common line $\mathbf{q}=\mathbf{e}_{i} \times \widetilde{\mathbf{e}}_{i}$ for which $l_{l_{i}}=0$ and due to the continuity near this map area low values can occur (see Figure $4(\mathrm{~d}-\mathrm{f})$ ). However, the maximum $\varsigma_{i}$ of $\varphi_{i}$

$$
\varsigma_{i}=\max _{\mathbf{x}_{i} \in I_{i}} \varphi_{i}\left(\mathbf{x}_{i}\right)
$$

can give a good indication about the precision of the matrix estimation $\widetilde{\mathrm{F}}$ with respect to the true fundamental matrix $\mathrm{F}$ when points from the image $I_{i}$ are projected to image $I_{i}$. The maximum $\varsigma$ on both images

$$
\varsigma=\max \left(\varsigma_{1}, \varsigma_{2}\right)
$$



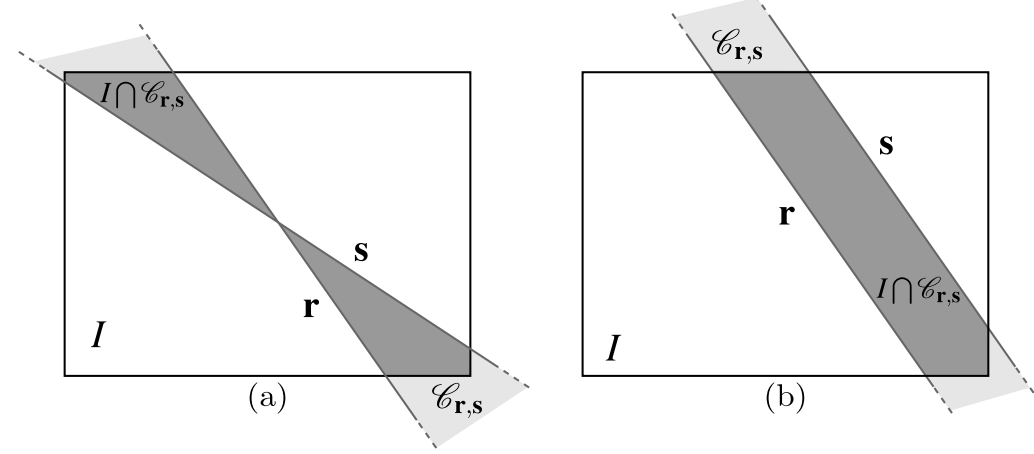

Figure 3: The cone $\mathscr{C}_{\mathbf{r}, \mathbf{s}}$ (grey) in the case the lines $\mathbf{r}$ and $\mathbf{s}$ intersect (a) or are parallel (b), and its intersection with the image $I$ (dark grey).

is finally used as error value. By considering the continuity of the map $\varphi_{i}$, in order to speedup the computation the $\varsigma_{i}$ values can be computed only on sampled points $\mathbf{x}_{i}$ homogeneously distributed on the image $I_{i}$, for instance on a grid.

\section{Evaluation}

\subsection{Experimental setup}

The new normalized epipolar error $\kappa_{i}$ has been compared against the Sampson error and the epipolar distance $d\left(\mathbf{x}_{i}, \mathbf{l}_{i}\right)$, applied to RANSAC, MSAC and MLESAC. To distinguish between them, the 'no' prefix is added in the case of the normalized epipolar error. In order to combine the error of both the images of the stereo pair in the case of epipolar errors $\varepsilon_{i} \in\left\{\kappa_{i}, d\left(\mathbf{x}_{i}, \mathbf{l}_{i}\right)\right\}$, three possible choices were considered, i.e. the symmetric epipolar error usually adopted in the case of the epipolar distance [10]

$$
\varepsilon_{S}=\frac{\varepsilon_{1}+\varepsilon_{2}}{2}
$$

the max epipolar error

$$
\varepsilon_{m}=\max \left(\varepsilon_{1}, \varepsilon_{2}\right)
$$

and the geometric epipolar error

$$
\varepsilon_{g}=\sqrt{\varepsilon_{1}^{2}+\varepsilon_{2}^{2}}
$$

A set of 10 sequences, made up of three images of a three-dimensional scene taken from different points of view, have been used, for a total of 30 stereo image pairs, four of which have been extracted from other works [26, 28, 32].

The HarrisZ keypoint detector [2] and the sGLOH descriptor [1], both proven to be robust and stable, have been used to get the matches ranked by a nearest neighbour selection. The former is based on the Harris corner detector [9], while the latter on the SIFT descriptor [12].

Different thresholds $t$ have been used. In the case of the normalized epipolar error, the whole range was tested, in particular 9 values from 0.1 to 0.9 by a step of 0.1 were investigated. It was found experimentally that a range from 0.5 to 4.5 pixels covers the best 


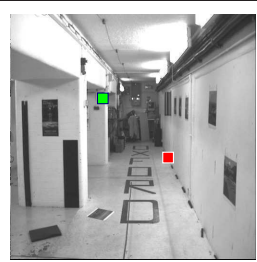

(a)

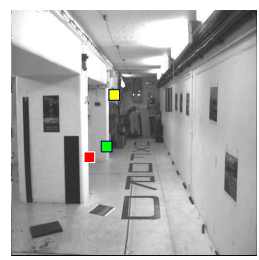

(d)

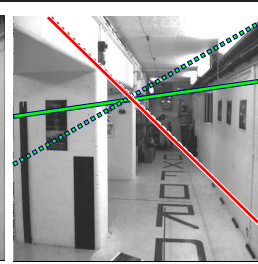

(b)

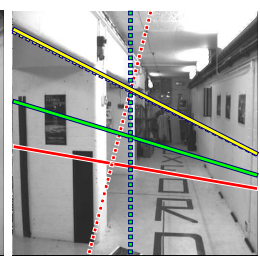

(e)

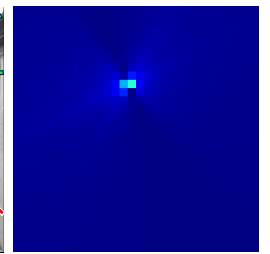

(c)

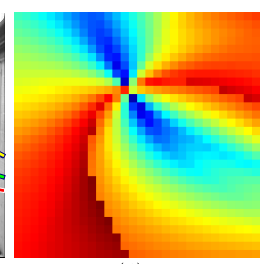

(f)

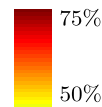

$25 \%$

$0 \%$

$\varphi_{i}$

Figure 4: Two different estimations of the same fundamental matrix. The points on the image $I_{i}(\mathrm{a}, \mathrm{d})$ correspond to the estimated (solid) and the true (dashed) epipolar lines on the other image $I_{\bar{i}}(\mathrm{~b}, \mathrm{e})$. Clearly the model on the top row (a-c) is better, as it is confirmed by inspecting the sampled maps $\varphi_{i}(\mathrm{c}, \mathrm{f})$. In the bottom row model (d-f) there is a discontinuity between the red and the green points, where the minimum angle made by the epipolar lines switches. When both the true and the estimated yellow epipolar lines pass through both the estimated and the true epipoles $\varphi_{i}\left(\mathbf{x}_{i}\right)=0 \%$.

threshold setting for the epipolar distance and the Sampson error. A step of 0.5 was used in this latter case to get 9 threshold values too.

In order to compare the methods, the $\varphi_{i}$ map was computed only on sampled points of the stereo pair images, which have size $m \times n$. The points have been sampled on an uniform Cartesian grid, where the stride $u$ is determined by $u=\min (m, n) / 30$.

For each stereo pair the ground truth fundamental matrix was computed by using the normalized eight-point algorithm [10] on more than 50 hand-taken correspondences, homogeneously distributed on the images. A maximum of 5000 iterations was set for each method and the final matrix estimation is computed again by the normalized eight-point algorithm on the best inlier set found by the method.

To take into account the randomness of RANSAC-based algorithms, the resulting $\varsigma$ error values are averaged on 30 runs for each possible choice of algorithm/threshold considered, for a total of $v=189$ different combinations, and the same random seed is used for each iteration among the different algorithm/threshold possibilities. Finally, for each stereo pair the different methods have been ranked according to the average of their errors $\varsigma$, named $\bar{\zeta}$.

\subsection{Result}

A global bar plot summarizing the results is reported in Figure 5. The global error of each method in the corresponding threshold range, $[0,1]$ for $\kappa_{i}$ and $[0,4.5]$ pixels for $d\left(\mathbf{x}_{i}, \mathbf{l}_{i}\right)$ is reported. For each stereo pair, the error $\bar{\zeta}$ for each method has been normalized by the mean and the standard deviation computed on the stereo pair itself, because the different errors are related to the complexity of the images which can vary with different stereo pairs. Next the 
final global error for a method is obtained by averaging the method errors on all the stereo pairs, shown in the plot normalized in the range $[0,1]$, higher bars means lower error values.

The error distribution can be retrieved also by looking at the different horizontal lines, which correspond to different error data percentiles, e.g. a value of 20 th $\%$ means the value of the sorted data in increasing order at position $20 \% \times v=20 \% \times 189=47$. More close are lines, denser is that data error region. Data after the 90 th \% error are not shown to improve the visualization. According to the error distribution, almost all methods provide similar results
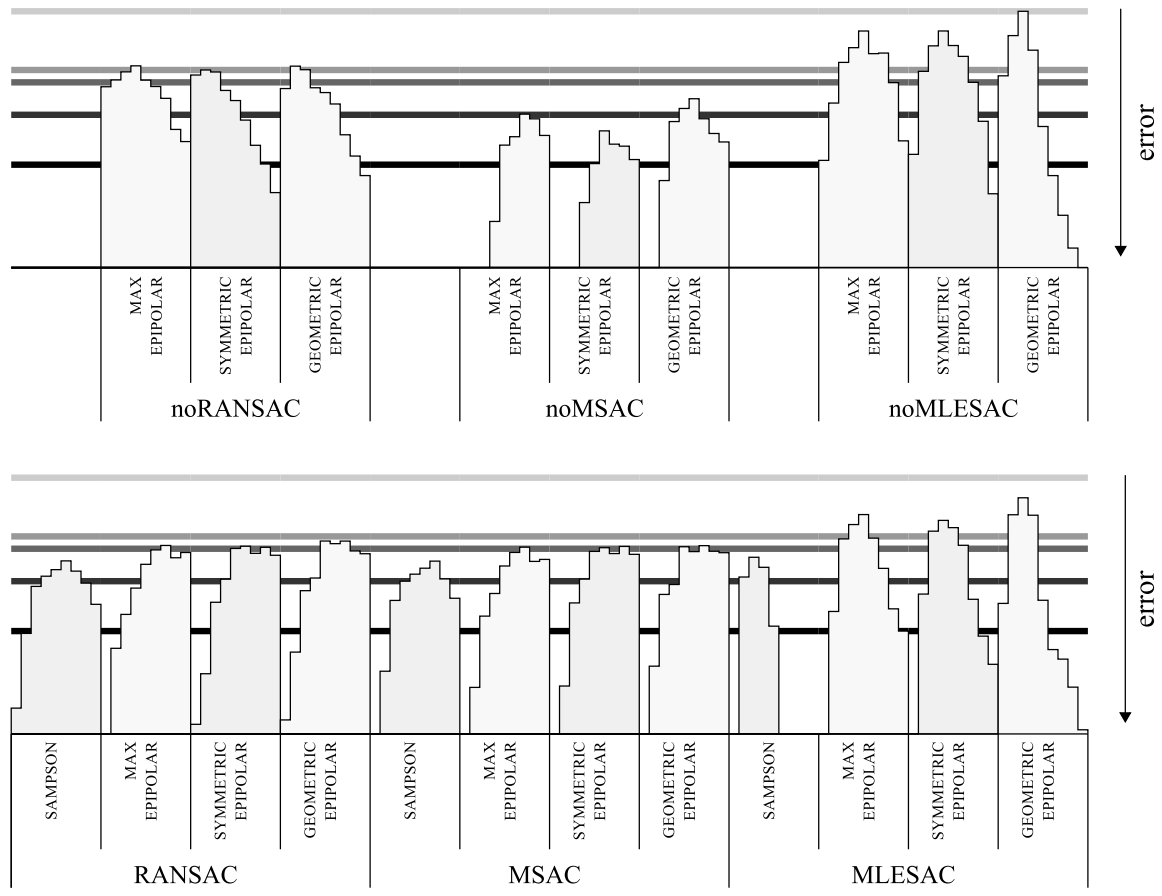

decreasing error rank $(\%)$

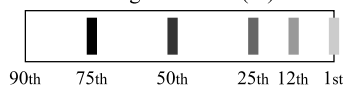

Figure 5: Global error for each methods. Higher bars indicate better results (see text).

since almost all error values are accumulated near the 25 th $\%$ rank position. As it can be seen, normalized RANSAC and MLESAC, namely noRANSAC and noMLESAC seems better than their original counterparts, while the opposite holds when comparing noMSAC with MSAC. Moreover, noMLESAC and MLESAC give the best results, followed by noRANSAC and RANSAC.

Furthermore, in the case of the normalized epipolar error the best threshold ranges around $[0.3,0.5]$, while this is different from RANSAC to MLESAC, as it can be seen from the position of the peaks in the plot. This is an experimental evidence that the normalized epipolar distance is more independent from both the method and the kind of images than its absolute counterpart, which implies higher stability and robustness of the proposed error measure.

As a further note, the geometric error $\varepsilon_{g}$ seems to provide the best results, while the Sampson error seems slightly less effective, opposing to what observed in [10]. 


\section{Conclusions and future works}

In this paper, a new normalized epipolar error measure is proposed to estimate the fundamental matrix with RANSAC-based approaches. The new distance makes use of data provided by the shape of the keypoints used as the point correspondences between the stereo image pair, with no further relevant computation.

In order to provide a comparison with existent error measures a novel evaluation strategy has been proposed too. It does not rely on the input matches but it indirectly employs a userprovided ground truth fundamental matrix, which can be obtained easily by a small number of hand-taken matches. No threshold-related issues are present, the results are more close to the required real solution and a meaningful measure is deduced.

According to the new evaluations strategy, the new normalized epipolar distance provides better results when applied to RANSAC or MLESAC and moreover it is more independent both from the applied method and from the input images, which makes it more robust and stable.

Future works will include to test the proposed normalized epipolar error to other RANSACbased approach on more input stereo pairs. About the evaluation strategy, further mathematical aspects can be investigated, as well as its application to the multiview geometry.

\section{Acknowledgments}

Thanks to Cesare Valenti, for the useful discussions and for his help in order to estimate the ground truth fundamental matrices used in the experimental evaluation.

This work has also been partially supported by a grant "Fondo per il Potenziamento della Ricerca del Dipartimento di Matematica e Applicazioni dell'Università degli Studi di Palermo".

\section{References}

[1] F. Bellavia, D. Tegolo, and E. Trucco. Improving SIFT-based descriptors stability to rotations. In International Conference on Pattern Recognition, 2010.

[2] F. Bellavia, D. Tegolo, and C. Valenti. Improving Harris corner selection strategy. IET Computer Vision, 5(2), 2011.

[3] T. Botterill, S. Mills, and R. Green. New conditional sampling strategies for speeded-up RANSAC. In British Machine Vision Conference, 2009.

[4] O. Chum, J. Matas, and J. Kittler. Locally optimized RANSAC. In Deutsche Arbeitsgemeinschaft für Mustererkennung Symposium for Pattern Recognition, pages 236-243, 2003.

[5] O. Chum, T. Werner, and J. Matas. Two-view geometry estimation unaffected by a dominant plane. In Computer Vision and Pattern Recognition, pages 772-779, 2005.

[6] R.J.M. den Hollander and A. Hanjalic. A combined RANSAC-hough transform algorithm for fundamental matrix estimation. In British Machine Vision Conference, 2007. 
[7] M.A. Fischler and R.C. Bolles. Random sample consensus: a paradigm for model fitting with applications to image analysis and automated cartography. Communications of the ACM, 24(6):381-395, 1981. ISSN 0001-0782.

[8] J.M. Frahm and M. Pollefeys. RANSAC for (quasi-)degenerate data (QDEGSAC). In IEEE Conference on Computer Vision and Pattern Recognition, pages 453-460, Washington, DC, USA, 2006. IEEE Computer Society. ISBN 0-7695-2597-0.

[9] C. Harris and M. Stephens. A combined corner and edge detector. In Alvey Vision Conference, pages 147-151, 1988.

[10] R. Hartley and A. Zisserman. Multiple View Geometry in Computer Vision. Cambridge University Press, 2000. ISBN 0-521-62304-9.

[11] A. Konouchine, V. Gaganov, and V. Veznevets. AMLESAC: A new maximum likelihood robust estimator. In International Conference on Computer Graphics and Vision, 2005.

[12] D.G. Lowe. Distinctive image features from scale-invariant keypoints. International Journal of Computer Vision, 60(2):91-110, 2004. ISSN 0920-5691.

[13] J. Matas and O. Chum. Matching with PROSAC - progressive sample consensus. In IEEE Conference on Computer Vision and Pattern Recognition. IEEE Computer Society, 2005. ISBN 0-7695-2372-2.

[14] J. Matas and O. Chum. Randomized RANSAC with sequential probability ratio test. In International Conference on Computer Vision, pages 1727-1732, 2005.

[15] A. Meler, M. Decrouez, and J.L. Crowley. BetaSAC: A new conditional sampling for RANSAC. In International Conference on Computer Vision, 2010.

[16] K. Mikolajczyk and C. Schmid. A performance evaluation of local descriptors. IEEE Transactions on Pattern Analysis and Machine Intelligence, 27(10):1615-1630, 2005.

[17] K. Mikolajczyk, T. Tuytelaars, C. Schmid, A. Zisserman, J. Matas, F. Schaffalitzky, T. Kadir, and L. Van Gool. A comparison of affine region detectors. International Journal of Computer Vision, 65(1-2):43-72, 2005. ISSN 0920-5691.

[18] D.R. Myatt, P.H.S. Torr, S.J. Nasuto, J.M. Bishop, and R. Craddock. NAPSAC: High noise, high dimensional robust estimation - it's in the bag. In British Machine Vision Conference, 2002.

[19] K. Ni, H. Jin, and F. Dellaert. GroupSAC: Efficient consensus in the presence of groupings. In International Conference on Computer Vision. IEEE Computer Society, 2009.

[20] D. Nistér. Preemptive RANSAC for live structure and motion estimation. In IEEE International Conference on Computer Vision, page 199, Washington, DC, USA, 2003. IEEE Computer Society. ISBN 0-7695-1950-4.

[21] D. Nistér. An efficient solution to the five-point relative pose problem. IEEE Transactions on Pattern Analysis and Machine Intelligence, 26(6):756-777, 2004. ISSN 0162-8828. 
[22] R. Raguram, J.M. Frahm, and M. Pollefeys. A comparative analysis of RANSAC techniques leading to adaptive real-time random sample consensus. In European Conference on Computer Vision, pages 500-513, Berlin, Heidelberg, 2008. Springer-Verlag. ISBN 978-3-540-88685-3.

[23] R. Raguram, J.M. Frahm, and M. Pollefeys. Exploiting uncertainty in random sample consensus. In International Conference on Computer Vision, pages 47-52, 2009.

[24] V. Rodehorst and O. Hellwich. Genetic algorithm sample consensus (GASAC) - a parallel strategy for robust parameter estimation. In International Workshop 25 Years of RANSAC, IEEE Computer Society, pages 1-8, New York, USA, June 2006.

[25] T. Sattler, B. Leibe, and L. Kobbelt. SCRAMSAC: Improving RANSAC's efficiency with a spatial consistency filter. In International Conference on Computer Vision, pages 2090-2097. IEEE Computer Society, 2009.

[26] N. Snavely. Bundler: Structure from motion (SfM) for unordered image collections, 2010. http://phototour.cs.washington. edu/bundler.

[27] N. Snavely, S.M. Seitz, and R. Szeliski. Modeling the world from internet photo collections. International Journal of Computer Vision, 80(2):189-210, 2008. ISSN 09205691.

[28] E. Tola. Local descriptor for dense wide-baseline stereo matching, 2010. http://cvlab.epfl.ch/ tola/daisy.html.

[29] P.H.S. Torr and A. Zisserman. MLESAC: a new robust estimator with application to estimating image geometry. Computer Vision and Image Understanding, 78(1):138156, 2000. ISSN 1077-3142.

[30] Z. Zhang. Determining the epipolar geometry and its uncertainty: A review. International Journal of Computer Vision, 27:161-195, April 1998. ISSN 0920-5691.

[31] Z. Zhang, R. Deriche, O. Faugeras, and Q. Luong. A robust technique for matching two uncalibrated images through the recovery of the unknown epipolar geometry. Artificial Intelligence, 78(1-2):87-119, 1995. ISSN 0004-3702.

[32] A. Zisserman et al. Oxford visual geometry group, 2010. http: //www.robots.ox.ac.uk/ vgg. 